\title{
'Preferential looking' for monocular and binocular acuity testing of infants
}

\author{
JANETTE ATKINSON, OLIVER BRADDICK, AND ELIZABETH PIMM-SMITH
}

From the Kenneth Craik Laboratory, University of Cambridge

SUMMARY A method is described for obtaining rapid and reliable estimates of acuity in infants, for both monocular and binocular viewing. The method depends on 'preferential looking', where the infant prefers to look at a striped pattern rather than a blank screen of matched mean luminance. A staircase procedure for testing is followed, with observations being recorded by a 'blind' observer (who does not know on which of the 2 screens the striped pattern is displayed). Monocular acuity estimates have been obtained for a group of infants 3 to 4 months old with normal refractions. Many of these infants show similar acuity values in the 2 eyes, with a few showing reliable differences between the eyes. To check reliability of the method a comparison of 2 independent interleaved staircase estimates of the same eye have been made. In general this check shows highly consistent estimates for a given eye of a given infant. Nearly all infants show slightly higher acuity estimates for binocular viewing than for monocular. The possible reasons for this difference are discussed. The clinical use of such a method is reported for a number of cases. The method has been found to be useful in a variety of clinical conditions where other available tests are not possible on young infants.

The forced-choice preferential looking (FPL) method is now well established as a means of estimating acuity and other visual detection performance in infants aged 1-6 months. ${ }^{1-5}$ The present authors have been using it routinely to assess clinical acuity deficits in this age range, as have others. Published work has generally used FPL with a binocularly viewing infant. However, clinical assessment is usually required for each eye separately. In many instances it is the possible difference between the eyes which is the major concern. Binocular relationships have also been the principal focus of recent work in the developmental neurobiology of vision, ${ }^{67}$ and therefore measurements of the performance of each eye separately are required if research on normal and abnormal visual development in infancy is to be related to these studies.

The study reported here was therefore intended to test whether reliable determination of monocular acuity was possible by FPL, what variation of acuity between the eyes would be found in a presumptively normal group of infants using this technique, and Correspondence to Department of Experimental Psychology. University of Cambridge. Downing Street. Cambridge CB2 3EB. whether the method would permit the detection of clinically significant interocular differences. As well as these trials of the method on normal infants we describe our experience in the practical use of the method for monocular and binocular testing of infants referred for acuity testing for a variety of clinical reasons.

\section{Material and methods}

The apparatus and general procedure resembled that described by Atkinson et $_{\text {al. }}{ }^{3}$ Infants were seated on a holder's knee $57 \mathrm{~cm}$ from a pair of oscilloscope screens, at which distance the diameter of each screen subtended $10.5^{\circ}$ and the separation of the screens $6 \cdot 3^{\circ}$. Between trials both screens displayed a uniform field of luminance $30 \mathrm{~cd} / \mathrm{m}^{2}$, and a set of lights midway between the screens attracted the infant's attention. A concealed observer watched the infant from a central peephole above the screens, and initiated a trial when the infant was fixating centrally. During the trial one of the screens (selected automatically at random) was modulated by a high-contrast horizontal sinusoidal grating while the other remained uniform and of equal mean luminance. (The gratings moved 
steadily with a temporal modulation rate of $3 \mathrm{~Hz}$.) The observer was unaware of which side the grating was displayed and had to make a forced choice of this based on observation of the infant's fixations and other behaviour. The duration of the observation period necessary for this was under the observer's control up to maximum of 10 seconds and was typically 5 seconds or less for infants of the age tested in this study. If on any trial an infant was not looking at either screen the observer could abandon that trial without having to make a forced-choice judgment.

For monocular testing an adhesive orthoptic patch was placed over the left or right eye. The observer checked the alertness and lack of fussiness of the infant by presenting a low spatial frequency grating $(0.5 \mathrm{c} / \mathrm{deg})$, and checking that the infant made a clear observable eye movement to the display on the side of the pattern. The infant's position in front of the screens was arranged so that before the pattern was presented the viewing eve was in line with the central fixation lights.

A series of spatial frequencies (grating stripe widths) were chosen in which each frequency was higher than the one below by a factor of 1.6 (twothirds octave). Testing was started with 5 trials using a grating of $2 \mathrm{c} / \mathrm{deg}$. After this the spatial frequency used for each block of 5 trials was selected according to a 'staircase' rule. ${ }^{8}$ That is, if on the previous block the observer had made 4 or 5 correct choices the spatial frequency was increased by one step for the next block. Two or fewer correct choices led to the next lowest spatial frequency being used in the next block. If there were 3 correct choices out of 5 , the frequency remained unchanged; if this occurred on 3 successive blocks, the next frequency was chosen in . the direction most likely to meet the criterion for completing the staircase.

A staircase was taken as complete when at least 20 trials had been run at each of 2 adjacent frequencies, with the proportion of correct responses bracketing or including the value of $70 \%$. Normally between 50 and 90 trials were needed to meet this criterion. The acuity value was taken as the $70 \%$ point obtained by interpolation between these 2 spatial frequencies.

To test acuity of the 2 eyes under conditions which were as comparable as possible in terms of the infant's state we used a method of 'interleaved staircases.' Twenty-five trials (i.e., 5 blocks) were run with one eye occluded; the occluding patch was then switched to the other eve and a new staircase sequence was followed for 25 trials.

The patch was then put back on the first eve. the next block of 25 trials followed the original staircase from where it had been left off, and so on. An example of the procedure is illustrated in Fig. 1. To assess the reproducibility of measurements made with our technique we tested some other infants with the same procedure of 2 interleaved staircases but with both independent staircase sequences testing the same eve. We shall refer to this as 'series $1 /$ series 2 comparison.'

The infants volunteered by their parents to help in this study were from the Cambridge area. All infants had normal birth histories and were not more than 10 davs premature. The mean age of testing on the first visit was 16 weeks $\left(3^{1 / 2}\right.$ months $)$. Testing on each

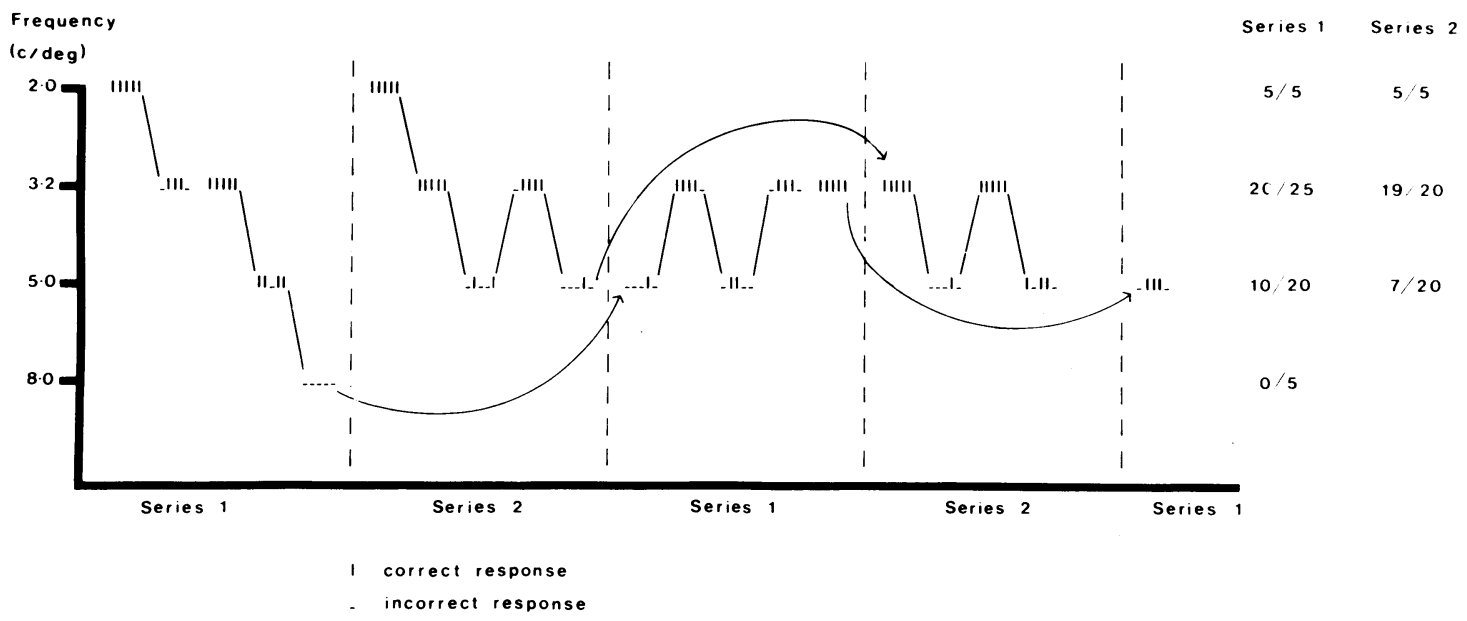

Fig.1 An example of the procedure used for infants who were tested with 2 independent staircases on one eve to obtain 2 interleaved estimates of acuity. 
child was completed within a period of 14 davs. To complete the testing most infants made 3 visits; a few completed testing in 2 visits and a few took up to 5 visits. They were tested when alert and calm, usually after a feed. No testing period was longer than 15 minutes.

All the infants were photorefracted under conditions of free accommodation ${ }^{9}$ to detect any refractive errors which might affect the comparisons being made. No infant showed any difference between the eyes greater than $0.5 \mathrm{D}$ in anv meridian.

Twenty-nine infants were tested in all, of whom 4 did not complete monocular testing because of fussiness and so are not included in the results. The remaining 25 received different conditions of testing in various sequences, outlined in the results section.

\section{Results}

Sixteen infants received interleaved staircases viewing with the left and right eyes. The acuity values obtained are indicated by solid circles on Fig. 2. This figure also includes data from 7 infants for whom each eye was tested but not in interleaved staircases. because they were initially in the group for series $1 /$ series 2 comparison (open circles on Fig. 2). In most cases measurements on the 2 eyes showed good agreement (i.e., the points lay close to the $45^{\circ}$ line). The median ratio of measured acuity between the eyes (ratios always taken as greater than $1 \cdot 0$ ) was $1 \cdot 12$. However, a few individuals showed a more marked interocular difference, which was as large as $2 \cdot 0$ in 2 cases.

To estimate the reliability of these figures we have the comparisons of series $1 /$ series 2 shown in Fig. 3. These data are from 6 infants for whom series $1 /$ series 2 were interleaved for one eye, and from each eve of 3 further infants for whom series $1 /$ series 2 were obtained on both eyes. (Data from the latter infants are included in Fig. 2 also.) All data points lav very close to the $45^{\circ}$ line (median acuity ratio for the two series $=1.06$; maximum ratio $=1 \cdot 20$ ). It appears therefore that the variations seen in Fig. 2 are not a consequence of variability in the method but reflect the range of genuine interocular differences at this age.

Fig. 4 shows the results of comparing binocular and monocular acuity determinations on the same infants. The monocular acuity shown was the better of the 2 values for each infant except in the 2 cases represented by open circles, in which only one eye received monocular testing. None of the binocular testing was interleaved with monocular testing. In general the estimated binocular acuities were higher than the better monocular acuities. There are several possible reasons for this difference between

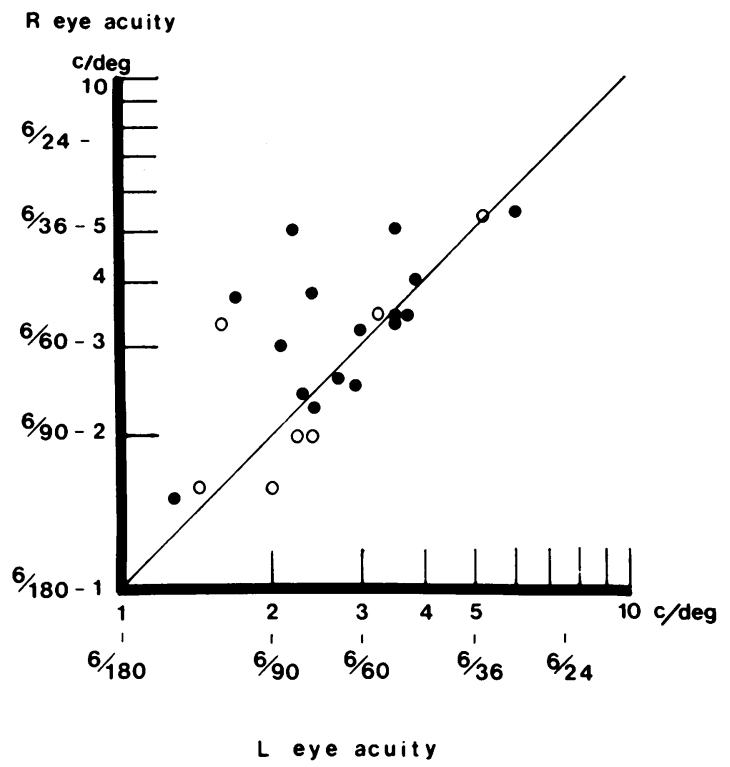

Fig. 2 Acuitv estimated for $R$ and $L$ eyes of 23 infants. The filled circles are infants where the 2 eyes' estimates were interleaved, the open circles are for noninterleaved results. Outer scales give Snellen equivalent of the acuity measures.

Monocular Acuity - repeated series on same eye acuity - Series 2

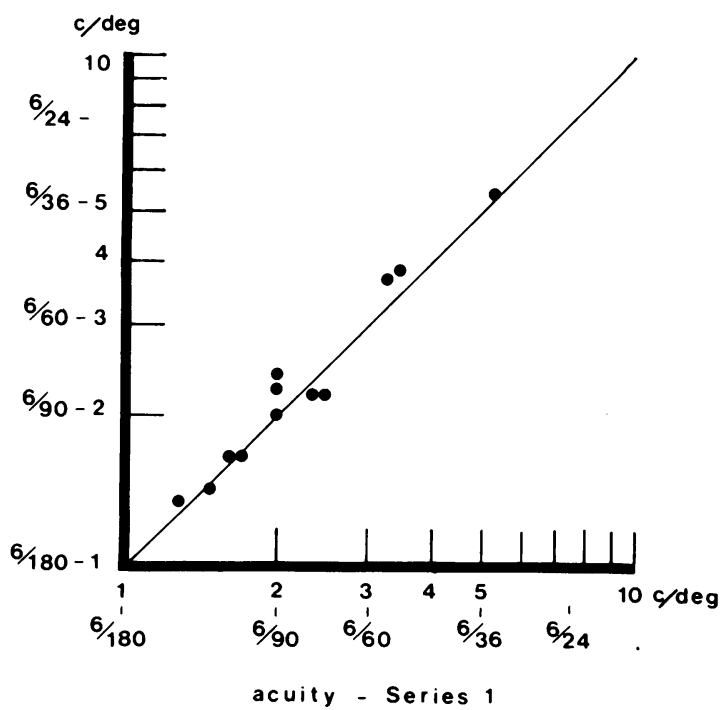

Fig. 3 Comparison of 2 acuity estimates with the same eye for 12 eyes from individual infants. The 2 estimates were interleaved in blocks of 25 trials. 


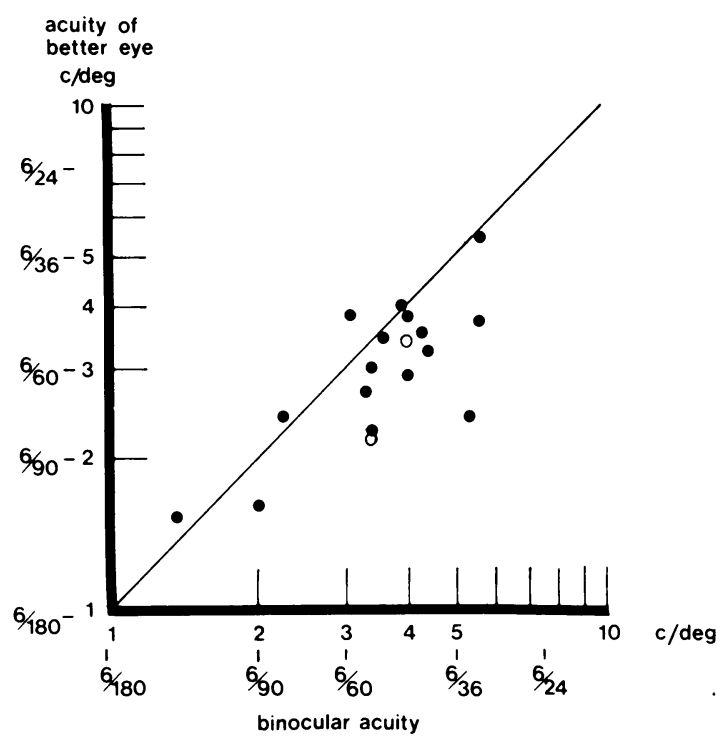

Fig. 4 Comparison of estimates of binocular acuity with the highest monocular acuity for 16 infants. The 2 open circles are for 2 infants for whom only one eye's acuity was estimated.

monocular and binocular results. It may be that infants' binocular resolution is genuinely better than monocular, as has been reported for adults. However, we do not believe that the data give strong evidence for such a sensory effect, since the infants were in an overall calmer state when they were not wearing a patch. While we made every attempt to conduct monocular and binocular testing with the infants in similar state and over a similar time, testing was usually more difficult when a patch was used. Further, as the binocular testing was on a later session than monocular, there may have been some binocular advantage from the rapid development of acuity at this age. This would be overcome by interleaved monocular and binocular testing.

CLINICAL USE OF THE METHOD

A number of clinical cases have been successfullv tested by using the procedure. It is hoped that some of these infants will be followed up by different tests when they are older.

In general the procedure has proved useful in assessing vision in 3 groups.

(1) Infants in whom there is a suspected problem in one eye. Infants in this group were tested monocularly as in the study reported above. The age of the infant appears to be the critical factor determining the possible length of testing. Infants older than 9 months were difficult to test monocularly and in general did not attend long enough to complete the staircase procedure. So far 41 infants have had monocular assessments of acuity. Ten of those had congenital convergent strabisimus, 7 of whom were tested before and after surgery. The other cases in this group were infants with high refractive errors (anisometropia, large oblique astigmatism requiring monocular testing with different grating orientations for the 2 eyes), corneal opacities or scarring, intermittent divergent strabismus, congenital cataract, nystagmus, coloboma, ptosis, microphthalmia, ophthalmia neonatorum. Many of the cases have had multiple assessments at different ages in the first year of life. A typical case is of a baby who was 8 weeks premature, with respiratory problems at birth, and intermittent left convergent strabismus. Tested at 6 months the LE acuity was $1 \cdot 2 \mathrm{c} / \mathrm{deg}(6 / 150)$, RE acuity $2 \cdot 5 \mathrm{c} / \mathrm{deg}(6 / 72)$.

Monocular assessments according to the procedure described were completed on 17 of these infants under 9 months of age and on 5 aged between 9 and 18 months. In 14 (all over 9 months) acuity estimates had to be derived from incomplete staircases, and in 5 cases the infants were not sufficiently co-operative for any useful testing.

(2) Infants with suspected poor sight, partial vision, or blindness. In general, if testing is binocular, a reliable assessment of acuity can be obtained for infants slightly older than when monocular assessment is required. The types of clinical disorders included in this group are large refractive errors, coloboma, hydrocephalus, cortical blindness, congenital nystagmus, microphthalmia, strabismus, birth trauma, and encephalitis. Out of 58 infants tested 14 under 9 months of age completed testing; 41 over 9 months of age had acuities estimated from abbreviated staircases (20-25 trials rather than $40-100$ trials), and 3 infants over 9 months of age were uncooperative.

A typical case in this group was a baby with coloboma of iris, lens, and choroid, tested at $2 \cdot 5$ months old, binocular acuity $1.0 \mathrm{c} / \mathrm{deg}(6 / 180) ; 5$ months, $3 \cdot 0 \mathrm{c} / \mathrm{deg}(6 / 60) ; 8$ months, $3 \cdot 0 \mathrm{c} / \mathrm{deg}(6 / 60)$.

(3) Children with developmental delay suspected of partial sight or blindness. In general these children are passive and easy to test because the response required from the child is minimal. To date 10 children in this category have been successfully tested; 6 of these children have delay of unknown aetiology, one child had severe tuberculous meningitis, one viral encephalitis, one intracranial haemorrhage at birth leading to hydrocephalus, and one agenesis of the corpus callosum.

An example of such a case was a baby aged $2 \cdot 5$ years with severe mental retardation (mental age approximately 4 months) and binocular visual acuity $4 \mathrm{c} / \operatorname{deg}(6 / 45)$. 


\section{Discussion}

The results of Figs. 2 and 3 show that reliable monocular estimates of acuity are possible with infants by the preferential looking (PL) method, although it demands more attention to infant handling than binocular use of the same method. PL is therefore suitable for clinical assessment of monocular as well as binocular disorders of infant vision. But we have also shown measurable differences in interocular acuity in presumptively normal 3-month-olds. The reason for these differences is not known. However, it is perhaps not surprising that in a period of rapid acuity development ${ }^{10}$ the 2 eyes might sometimes be found to be at slightly different stages of that development. We presume that such differences are transient. Acuity differences of a factor of more than 2, or differences that persist, may be considered as probable indications of abnormality.

In the PL procedure to assess acuity in clinical cases we find it more successful in infants under 9 months (or equivalent developmental age) than in older infants. Estimates are often possible for the older infants, but it is frequently not possible to maintain the infant's attention long enough to complete the staircase procedure described here. We hope that it may prove possible to apply more sophisticated statistical procedures to PL testing ${ }^{1112}$ and thereby reduce the number of trials necessary to achieve a reliable threshold estimate.

There is still a great need for a robust test suitable for children over 9 months but too young for subjective tests such as the Sheridan-Gardiner. The tracking tests such as Catford drum ${ }^{13}$ and Stycar balls ${ }^{14}$ suffer from some shortcomings in target design and procedure. ${ }^{15} \mathrm{We}$ are currently exploring the application of tests which depend on tracking but use stimuli that are genuine resolution targets, and which maintain the principles of forced choice by a blind observer and staircase determination of threshold which we have applied in the PL test.
This work was supported by the Medical Research Council. We would like to thank our clinical colleagues from the Department of Ophthalmology (especiallv Mr P. G. Watson and Mr J. Keast-Butler) and the Department of Paediatrics. Addenbrooke's Hospital. Cambridge, for referring their infants to us and for helpful discussions of the results.

\section{References}

1 Teller DY. Morse R. Borton R. Regal D. Visual acuity for vertical and diagonal gratings in human infants. Vision Res 1974; 14: $1433-9$.

2 Teller DY. The forced-choice preferential looking procedure: a psychophysical technique for use with human infants. Infant Behav Devel 1979; 2: 135-53.

3 Atkinson J. Braddick O. Moar K. Development of contrast sensitivity over the first 3 months of life in the human infant. Vision Res 1977; 17: 1037-44.

4 Banks MS. Salapatek P. Acuity and contrast sensitivity in 1-. 2-. and 3-month-old human infants. Invest Ophthalmol Visual Sci 1978: 17: 361-5.

5 Gwiazda J. Brill S. Mohindra I. Held R. Infant visual acuity and its meridional variation. Vision Res 1978; 18: 1557-64.

6 Hubel DH. Wiesel TN. Functional architecture of macaque monkey visual cortex. Proc R Soc Lond (Biol) 1977: 198: 1-59.

7 Blakemore C. Maturation and modification in the developing visual pathwav. In: Held R. Leibowitz HW. Teuber HL. eds. Handbook of Sensory Physiology: Perception. Berlin: Springer. 1978: 8.

8 Cornsweet TN. The staircase method in psychophysics. Am J Psvchol 1962: 75: 485-91.

9 Braddick OJ. Atkinson J. French J. Howland HC. A photorefractive study of infant accommodation. Vision Res 1979; 19: 1319-30.

10 Dobson V. Teller DY. Visual acuity in human infants: a review and comparison of behavioural and electrophysiological studies. Vision Res 1978; 18: 1469-83.

11 Taylor MM. Creelamn CD. PEST: Efficient estimates on probability functions. J Acoust Soc Am 1967; 41: 782-7.

12 Watson AB. Pelli DG. The Quest staircase procedure. AVA Newsletter 1979: no. 14.

13 Catford GV. Oliver A. Development of visual acuity. Arch Dis Child 1973; 48: 47-50.

14 Sheridan MD. Manual for the STYCAR vision tests. NFER Publishing Company, 1976.

15 Braddick OJ. Atkinson J. Pimm-Smith E. Ayling L. Sawyer R. Does the Catford drum give an accurate assessment of acuity? $\mathrm{Br}$ J Ophthalmol 1981: 65: 652-6. 